\title{
Etude ethnobotanique de quelques lavandes marocaines spontanées
}

\author{
Lamiae BACHIRI $^{1,2^{*}}$, Nadia LABAZI ${ }^{1,2}$, Amine DAOUDI $^{1}$, Jamal IBIJBIJEN $^{1}$, \\ Laila NASSIRI $^{1}$, Ghizlane ECHCHEGADDA ${ }^{2}$ et Faouzia MOKHTARI ${ }^{1}$ \\ ${ }^{l}$ Equipe de Microbiologie du Sol et de l'Environnement, Université Moulay Ismail, \\ Faculté des Sciences, Meknès, Maroc. \\ ${ }^{2}$ Département de la Protection de Plantes et de l'Environnement, \\ Ecole Nationale d'Agriculture, Meknès, Maroc. \\ *Auteur correspondant ; E-mail : lamiaebachiri@gmail.com ; \\ B.P : 11201, Meknès, Maroc. Tel : (+212) 618859436, (+212)14561720
}

\section{RESUME}

Le genre Lavandula est l'un des plus importants genres de la famille des Lamiacées et est riche en plantes à usage multiple, alimentaire, fourrager, aromatique, cosmétique et médicinal. Au Maroc, la bibliographie fait allusion à plusieurs espèces de ce genre, communément appelées lavandes, à écologie et phytogéographie diversifiées et dont d'une part la distinction taxinomique n'est pas des plus faciles et d'autre part l'éventualité d'un usage différent selon les localités et ou les espèces n'est pas investie. Aussi, l'objectif du présent travail est de rechercher les lavandes dans différentes régions du Maroc; puis, par le biais d'investigations ethnobotaniques auprès d'herboristes et de vendeurs de plantes aromatiques et médicinales (PAM), inventorier leurs principaux usages traditionnels. Ainsi, durant l'année 2014, des prospections floristiques à la recherche de lavandes sauvages ont permis de repérer et localiser Lavandula stoechas $L$ et L. atlantica Braun-Blanq, L. pedunculata Mill, L. multifida L, L. dentata L, L. maroccana Murb respectivement au plateau central « Oulmès, et Aguelmous » et au moyen atlas central « Ait Amro Ouali », la plaine de Sais «Ain Jerri », et en haut atlas occidental « bassin versant d'Ourika ». Les enquêtes ethnobotaniques menées auprès d'herboristes et vendeurs dans chaque site de récolte, via un questionnaire préétabli ont permis de dresser la liste des noms vernaculaires utilisés pour chaque espèce et ont révélé que les lavandes ont essentiellement des vertus thérapeutiques, que les feuilles et les fleurs sont les parties les plus utilisées surtout sous forme de décoction pour le traitement d'affections broncho-pulmonaires, gastrointestinales et uro-génitales.

(C) 2015 International Formulae Group. All rights reserved.

Mots clés : Lavandula, plantes aromatique et médicinales, enquête ethnobotanique, Maroc.

\section{INTRODUCTION}

En dépit du grand développement de l'industrie pharmaceutique, environ $80 \%$ de la population mondiale tire encore profit de la médecine traditionnelle reconnaissant par là les savoirs empiriques des ancêtres (EL Rhaffari et Zaid, 2004).

Ainsi, en Afrique, la médecine traditionnelle à base de plantes est dans de nombreuses situations la seule source de soins 
de santé surtout pour les populations habitant l'arrière-pays (Kitwa et Malebo, 2004).

Le Maroc jouit d'une position géographique privilégiée qui lui offre une grande diversité climatique et édaphique, lui conférant en l'occurrence des conditions favorables pour le développement d'une flore variée et riche de quelques 600 espèces aromatiques et médicinales (Hmamouchi, 2001). Par ailleurs, le Maroc constitue en méditerranée, un véritable réservoir phytogénétique ; il est l'un des pays à posséder une longue tradition médicale et un savoirfaire ancestral à base de plantes médicinales (Hmamouchi, 2001), parmi lesquelles on trouve de nombreuses espèces de la famille des lamiacées. En effet, la plupart des plantes de cette famille constituent une source d'huiles essentielles et sont très utilisées en aromathérapie, parfumerie et en industrie des cosmétiques (Benabdelkader, 2014). Tel est le cas des espèces appartenant aux genres Thymus, Rosmarinus, Salvia, Mentha, Lavandula; ce dernier taxon est affilié à la sous-famille des Népétoïdées (Dupont et Guignard, 2012) et est composé d'environ 39 espèces, de nombreux hybrides, et près de 400 cultivars enregistrés (Upson et Anderws, 2004).

Le présent travail est consacré d'abord à la recherche et la localisation de différentes lavandes sauvages marocaines, puis, par le biais d'investigations ethnobotaniques auprès d'herboristes et vendeurs de plantes aromatiques et médicinales (PAM), inventorier leurs principaux usages.

\section{MATERIEL ET METHODES}

Prospection, recherche et identification des espèces

Des prospections floristiques ont été menées dans différentes régions du Maroc (Tableau 1) dans le but de rechercher et localiser les lavandes sauvages; les échantillons récoltés furent ensuite identifiés par une équipe de botanistes de la faculté des
Sciences, de l'université Moulay Ismail à Meknès.

Au niveau de chaque site, une enquête ethnobotanique a été menée auprès de vendeurs et d'herboristes via un questionnaire préétabli comportant en plus de questions se rapportant à l'enquêté lui-même, des questions précises quant au nom local (vernaculaire) de chaque espèce, la partie utilisée, les modes de préparation et les usages thérapeutiques.

\section{Analyse statistique}

Le traitement statistique des données brutes a été réalisé à l'aide du logiciel SPSS (17.0) pour une meilleure exploitation des données récoltées et une bonne présentation des résultats obtenus.

\section{RESULTATS}

Identification et exigences climatiques et édaphiques des lavandes récoltées

Les sites où la lavande fut repérée à l'état sauvage dans cette étude sont reportés sur la carte du Maroc (Figure 1).

Les échantillons récoltés ont été identifiés sur la base de plusieurs critères, notamment la morphologie et la couleur des feuilles, la forme de l'inflorescence et celle des bractées, surtout les fertiles « axillant des fleurs » (Tableau 2).

Par ailleurs une quête d'informations auprès du personnel des eaux et forêts et des Directions Provinciales d'Agriculture (DPA), supplée de documentation principalement, la flore du Maroc et appuyée d'observations sur le terrain nous a permis de dresser un tableau résumant aussi bien les exigences climatiques qu'édaphiques des différentes espèces identifiées (Tableau 3).

\section{Résultats de l'enquête ethnobotanique Sexe et niveau d'instruction des enquêtés}

La plupart des vendeurs de lavandes et des herboristes sont de sexe masculin, respectivement $90 \%$ et $72 \%$ (Figure 2). 
Par ailleurs, les différentes enquêtes ont révélé que la majorité des herboristes et vendeurs de lavande sont analphabètes (56 et $57 \%$ ) ; toutefois, certains d'entre eux ont quand même un niveau universitaire $(7 \%$ et $13 \%)$ respectivement.

\section{Nom local des lavandes étudiées}

L'investigation ethnobotanique dans les différentes localités où des lavandes spontanées ont été repérées a montré que le nom local «Lhalhal » est le même pour $L$. pedunculata, L. stoechas et L. atlantica alors que les espèces L. marrocana, L. dentata et $L$. multifida ont chacune un nom vernaculaire propre, respectivement, Timzouria, hlhal marakchiya et Kohyla.

\section{Les usages de la plante selon les localités}

La présente étude a montré que la lavande est utilisée essentiellement en thérapie (67.7\%) en comparaison avec la cosmétique $(19.4 \%)$ et autres «alimentation de bétail, aromatisant d'intérieur...» (12.9\%) (Tableau 4).Toutefois, certaines spécificités locales persistent; par exemple, à Ras Bariakh, la lavande est utilisée uniquement en phytothérapie (100\%).

Parties de la plante utilisées et modes de préparation

Les parties végétales à usage thérapeutique sont variées ; dans cette étude, il ressort que les parties utilisées sont la feuilles et les fleurs ; ces deux organes sont utilisés soit seuls ou ensemble (Figure 4). Cependant, si l'utilisation concomitante des deux parties est commune à toutes les localités étudiées, le recours aux deux parties séparément ne se fait pas à Ras Bariakh et à Ain Jerri ; de son côté, le recours à la feuille seule est absent à Douar Akhlij (Figure 4).

D'un autre côté, plusieurs modes de préparation sont employés à savoir, la décoction, l'infusion, la macération et le cataplasme ; toutefois, la majorité des remèdes est préparée sous forme de décoction (Figure 5) ; celle-ci est même le seul mode utilisé à Ras Bariakh (100\%). Aussi, selon les habitudes des usagers de ces plantes au niveau des différentes localités investies, ce mode de préparation permettrait l'élimination des substances toxiques et donc les risques d'intoxication.

Cependant, certaines différences sont à relever entre les localités et donc les espèces ; si la décoction paraît commune à toutes les localités, le cataplasme est signalé uniquement pour l'espèce L. stoechas à Oulmès et la macération, en haut Atlas pour L. dentata (bassin de l'Amassine) et L. maroccana (Douar Akhlij) ; enfin, la lavande d'Atlas (Ras Bariakh) est utilisée uniquement en décoction (Figure 5).

\section{Les pathologies traitées}

Les résultats obtenus montrent que les gens ont recours à la lavande pour traiter diverses affections telles celles uro-génitales, broncho-pulmonaires et gastro-intestinales ainsi que pour calmer les douleurs rénales et rhumatismales (Figure 6).

Toutefois, une certaine différence est relevée selon la région étudiée et donc selon le type de lavande qui y pousse; ainsi, à Ras Bariakh, là où domine L. atlantica, celle-ci est vendue uniquement pour le traitement d'affections gastro-intestinales (100\%); pareillement, à Aïn Jerri, L. multifida y est vendue pour le traitement des seules affections broncho-pulmonaires (100\%) (Figure 6). Dans ce sens, c'est pour la lavande stéchade que l'usage est le plus large couvrant toutes les affections auparavant citées à l'exception du rhume. Enfin, le recours à la lavande en cas de douleurs rénales n'est mentionné que pour la lavande dentée dans le bassin de l'Amassine (Figure 6). 


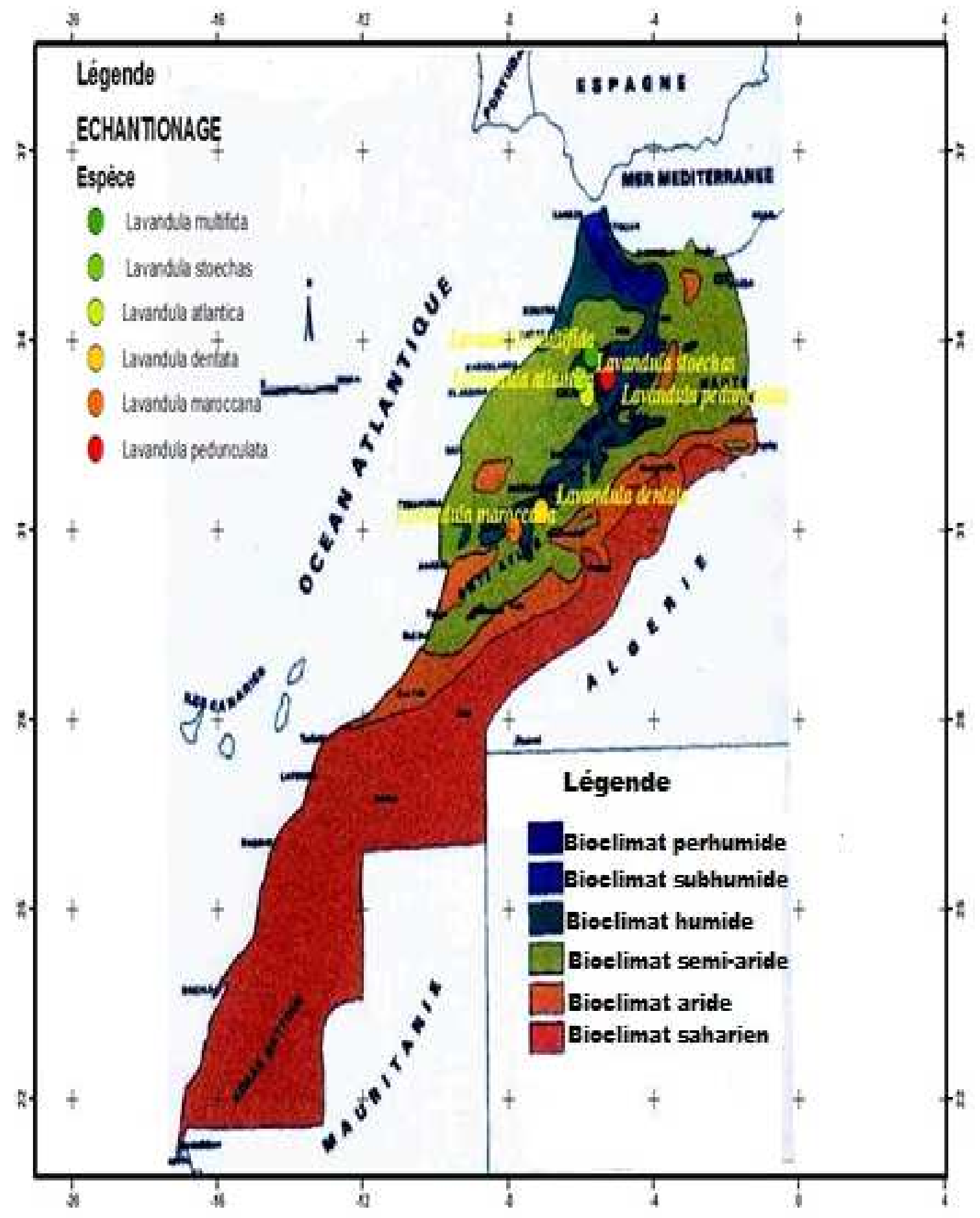

Figure 1 : Localisation des sites de récolte de lavandes. 
Tableau 1 : Lieu, date et période d'échantillonnage de lavandes.

\begin{tabular}{|c|c|c|c|}
\hline Espèce (E) & Localités & Date de récolte & Période de récolte \\
\hline E1 -E2 & $\begin{array}{ll} & \text { Plateau central : } \\
\text { 1- } & \text { Ras Bariakh (Aguelmous) } \\
& \text { 2- Oulmès }\end{array}$ & $\begin{array}{l}30 / 05 / 2014 \\
23 / 03 / 2014\end{array}$ & Pleine floraison \\
\hline $\mathbf{E 3}$ & $\begin{array}{l}\text { - } \quad \text { Moyen Atlas central : } \\
\text { 3- } \quad \text { Ait Amro Ouali (Azrou) }\end{array}$ & $13 / 04 / 2014$ & Pleine floraison \\
\hline E4-E5 & $\begin{array}{c}\text { - Haut Atlas occidental : } \\
\text { 4- } \quad \text { Douar Akhlij (Bassin versant de } \\
\text { l'Ourika) } \\
\text { 5- } \quad \text { Vallée de l'Amassine (Bassin versant } \\
\text { de l'Ourika) }\end{array}$ & $\begin{array}{l}17 / 04 / 2014 \\
18 / 04 / 2014\end{array}$ & Pleine floraison \\
\hline E6 & $\begin{array}{c}\text { Plaine de Saiss : } \\
\text { 6-Aïn Jerri (Meknès) }\end{array}$ & $08 / 05 / 2014$ & Pleine floraison \\
\hline
\end{tabular}
Lavandula maroccana, E5: Lavandula dentata spp.dentata, E6: Lavandula multifida .

Tableau 2 : Critères morphologiques distinctifs des espèces de lavande récoltées.

\begin{tabular}{|c|c|c|c|}
\hline Espèces & Feuille & Bractée & Inflorescence \\
\hline $\begin{array}{l}\text { E1:L. } \\
\text { pedunculata } \\
\text { spp.atlantica }\end{array}$ & $\begin{array}{l}\text { - Simples, entières, } \\
\text { lancéolées } \\
\text { de 3-4 cm, couleur vert- } \\
\text { grisâtre }\end{array}$ & $\begin{array}{c}\text { - Les fertile } \\
\text { circulaires, } \\
\text { largement ob- } \\
\text { ovales et arrondies; } \\
\text {-Les stériles brèves }\end{array}$ & $\begin{array}{l}\text {-De } 3-4 \mathrm{~cm} \text { de long à } \\
\text { fleurs bleu- violet portées } \\
\text { par un pédoncule d'une } \\
\text { dizaine de } \mathrm{cm}(3-10-\mathrm{cm})\end{array}$ \\
\hline $\begin{array}{l}\text { E2: L. stoechas } \\
\text { spp. Stoechas }\end{array}$ & $\begin{array}{l}\text {-Simples, entières de } 2-3 \mathrm{~cm} \\
\text { de long, lancéolées et de } \\
\text { couleur vert-grisâtre }\end{array}$ & $\begin{array}{l}\text {-Les fertiles, de } \\
\text { couleur violette, } \\
\text { largement ovales et } \\
\text { brièvement } \\
\text { acuminées; } \\
\text {-les stériles très } \\
\text { saillantes valant à } \\
\text { l'espèce le nom de } \\
\text { lavande papillon }\end{array}$ & $\begin{array}{l}\text {-Compacte, à pédoncule } \\
\text { court et moins long que la } \\
\text { masse florale ; les fleurs } \\
\text { sont de couleur violet } \\
\text { foncé }\end{array}$ \\
\hline $\begin{array}{l}\text { E3: L. } \\
\text { pedunculata spp. } \\
\text { pedunculata }\end{array}$ & $\begin{array}{c}\text {-Simples, entières de } 2-4 \mathrm{~cm} \\
\text { de long tomenteuses } \\
\text { oblongues }\end{array}$ & $\begin{array}{l}\text {-Les fertiles ob- } \\
\text { ovales, arrondies et } \\
\text { apiculées }\end{array}$ & $\begin{array}{l}\text {-Fleurs violettes à bleu- } \\
\text { violettes de } 2-3 \mathrm{~cm} \text { de } \\
\text { long, portées par des } \\
\text { pédoncules longs de } 10 \text { - } \\
20 \mathrm{~cm}\end{array}$ \\
\hline E4: L. maroccana & $\begin{array}{c}\text {-Pennées de } 2,5 \text { à } 3 \mathrm{~cm} \\
\text { ovales-lancéolées }\end{array}$ & $\begin{array}{l}\text {-Les fertiles à } \\
\text { forme orbiculaire- } \\
\text { pentagonale avec } \\
\text { un apex mucroné } \\
\text { ou acuminé }\end{array}$ & -Epis longs de $3-4 \mathrm{~cm}$ \\
\hline
\end{tabular}




\begin{tabular}{lccc}
\hline $\begin{array}{l}\text { E 5: L. dentata } \\
\text { spp.dentata }\end{array}$ & $\begin{array}{c}\text {-Entières crénelées; } \\
\text { grisâtres en dessous, vertes } \\
\text { en dessus, avec des poils } \\
\text { courts et peu denses }\end{array}$ & $\begin{array}{c}\text {-Les fertiles, } \\
\text { largement ob- } \\
\text { ovales de couleur } \\
\text { bleuâtre, }\end{array}$ & $\begin{array}{c}\text {-Epis de fleurs bleues - } \\
\text { violacées }\end{array}$ \\
\hline E 6: L. multifida & $\begin{array}{c}-3-6 \mathrm{~cm} \text { de long, } \\
\text { multifides, pétiolées et } \\
\text { pennatiséquées. }\end{array}$ & $\begin{array}{c}\text {-Les fertiles ovales } \\
\text { et subégales au } \\
\text { calice, larges, à } \\
\text { apex brusquement } \\
\text { aigu }\end{array}$ & $\begin{array}{c}\text {-Epis ne dépassant pas 3 } \\
\text { cm et à fleurs violacées }\end{array}$ \\
& & & \\
\hline
\end{tabular}

Tableau 3 : Exigences Climatiques et édaphiques des différentes espèces récoltées.

\begin{tabular}{lcc}
\hline Espèces & Ambiance bioclimatique & Nature du sol \\
\hline $\begin{array}{l}\text { Lavandula pedunculata } \\
\text { spp.atlantica }\end{array}$ & Subhumide & $\begin{array}{c}\text { Argilo-limoneux, faible } \\
\text { profondeur, forte charge en } \\
\text { éléments schisteux -gréseux }\end{array}$ \\
\hline $\begin{array}{l}\text { Lavandula stoechas } \\
\text { spp. stoechas }\end{array}$ & Subhumide & Siliceux \\
\hline $\begin{array}{l}\text { Lavandula pedunculata spp. } \\
\text { pedunculata }\end{array}$ & Subhumide & Calcaire \\
\hline Lavandula maroccana & Semi-aride & Argilo-limoneux \\
\hline Lavandula dentata spp.dentata & Semi-aride & Argile rouge et pélites \\
\hline Lavandula multifida & Semi-aride, Subhumide & Calcaire \\
\hline
\end{tabular}

Tableau 4 : Usages des lavandes selon les enquêtés dans chacune des localités étudiées.

\begin{tabular}{lccc}
\hline \multirow{2}{*}{ Localités } & \multicolumn{3}{c}{ Usages de la plante en \% } \\
\cline { 2 - 4 } & Thérapeutique & Cosmétique & Autre \\
\hline Ras bariakh & $100,0 \%$ & $0 \%$ & $0 \%$ \\
Oulmès & $50,0 \%$ & $33,3 \%$ & $16,7 \%$ \\
Ait Amro Ouali & $62,5 \%$ & $25,0 \%$ & $12,5 \%$ \\
Douar Akhlij & $80,0 \%$ & $0 \%$ & $20,0 \%$ \\
Bassin de l'Amassine & $60,0 \%$ & $30,0 \%$ & $10,0 \%$ \\
Ain Jerri & $85,7 \%$ & $0 \%$ & $14,3 \%$ \\
Total (localités confondues) & $\mathbf{6 7 . 7 \%}$ & $\mathbf{1 9 . 4 \%}$ & $\mathbf{1 2 . 9 \%}$ \\
\hline
\end{tabular}




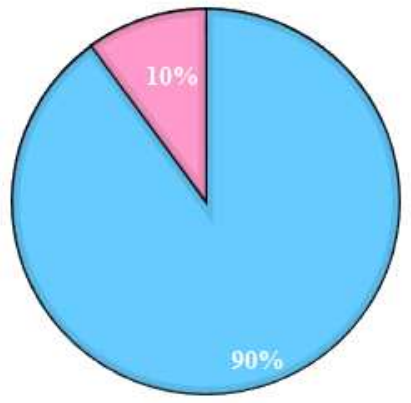

(a)
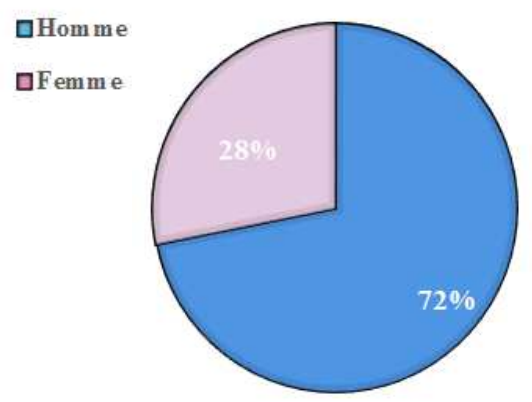

(b)

Figure 2 : Répartition des enquêtés selon le sexe (a : Vendeurs ; b : Herboristes).

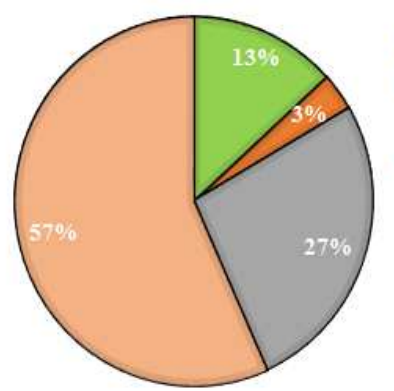

(a)
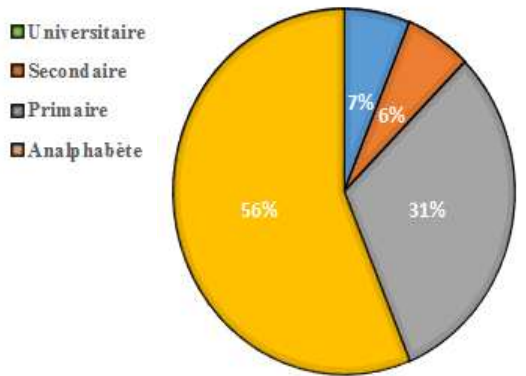

(b)

Figure 3 : Niveau d'instruction des enquêtés (a : Vendeurs ; b : Herboristes).

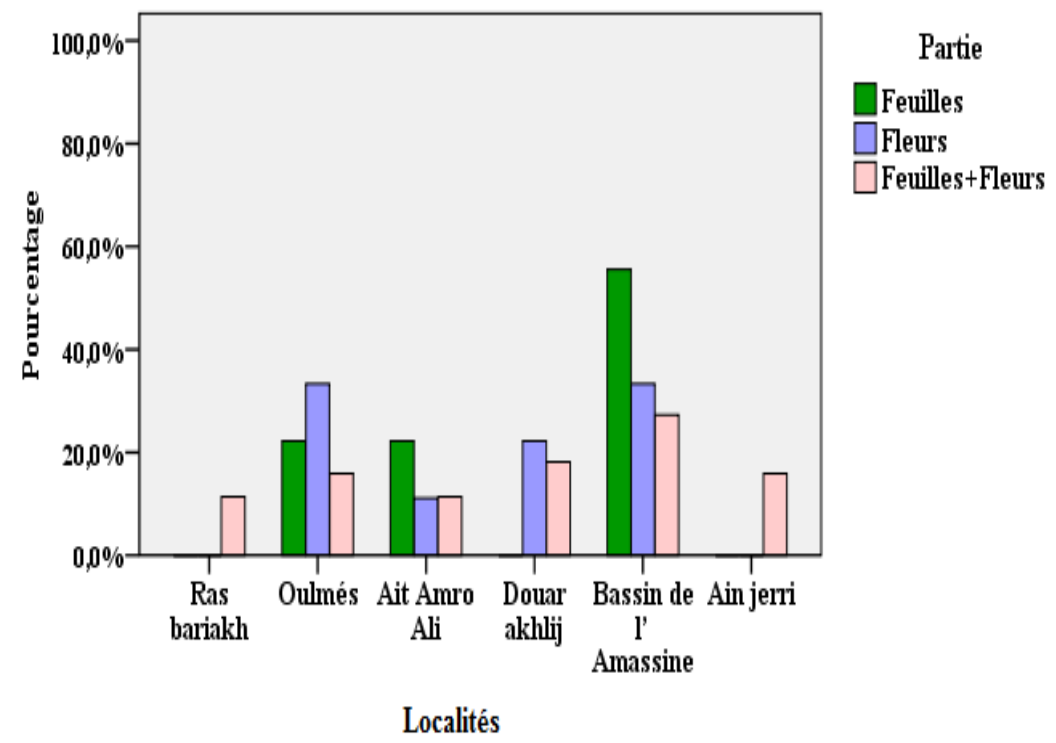

Figure 4 : Parties de lavandes utilisées en thérapie. 


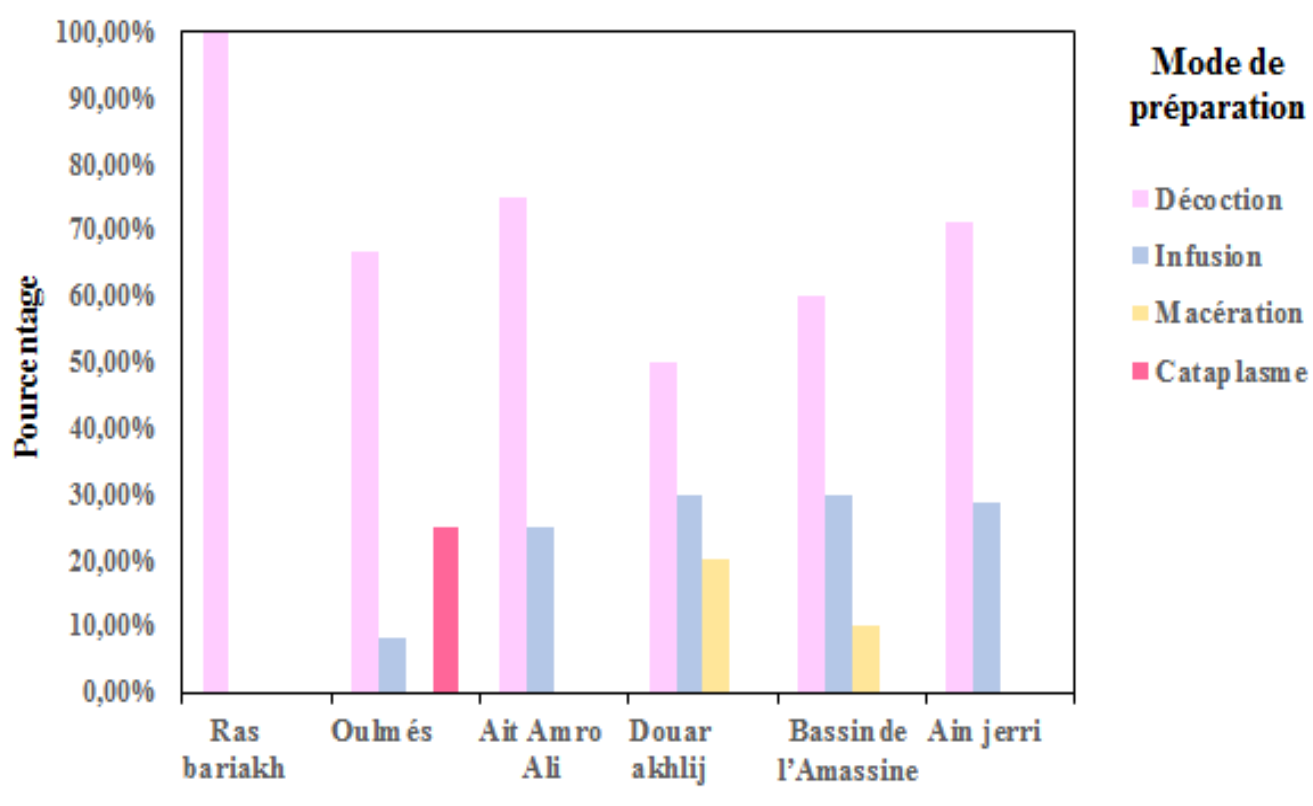

\section{Localités}

Figure 5 : Modes de préparation des remèdes à base de lavandes.

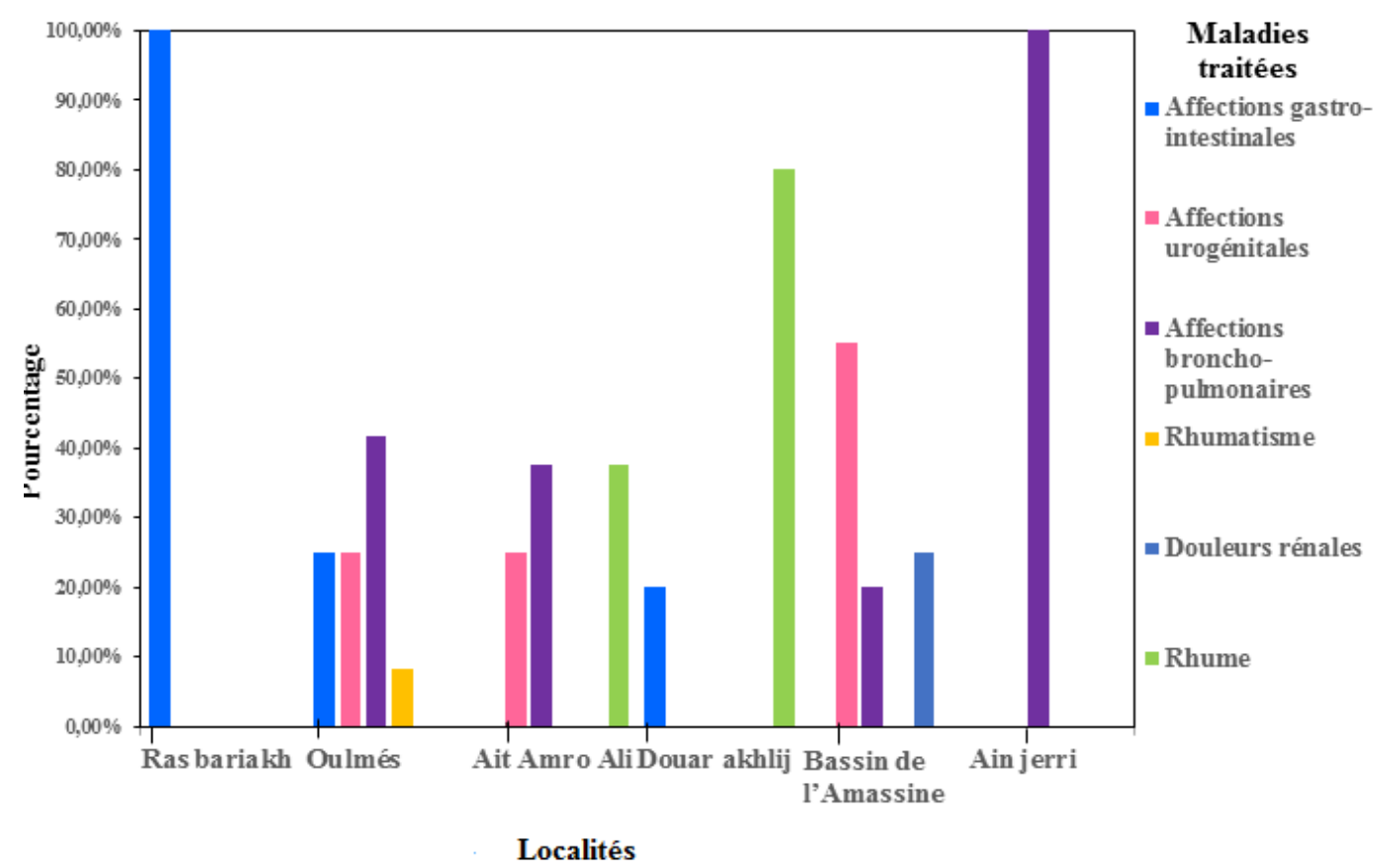

Figure 6 : Maladies traitées par les lavandes. 


\section{DISCUSSION}

Sexe et niveau d'instruction des enquêtés

Les résultats obtenus montrent que le secteur des PAM reste majoritairement un domaine d'hommes assez âgés ; en effet, les personnes les plus âgées ont plus de connaissances sur les propriétés et usages des espèces étudiées, suite à une longue expérience accumulée et transmise d'une génération à l'autre. Les jeunes par contre ne portent pratiquement aucun intérêt à l'usage thérapeutique des plantes et ont tendance à ne plus croire en la médecine traditionnelle, d'où l'importance des investigations ethnobotaniques pour sauvegarder cet héritage ancestral.

Par ailleurs, le taux d'analphabétisme, élevé parmi les acteurs des PAM constitue un handicap qui risque d'entraver l'évolution de la médecine traditionnelle au Maroc. Il devient donc impératif d'élaborer une stratégie cohérente, s'inscrivant dans le cadre de la stratégie nationale et en concertation avec différents acteurs (Botanistes, Sociologues, Chimistes, Taxonomistes et Anthropologues) pour une gestion rationnelle de ce domaine.

\section{Nom local des lavandes étudiées}

Les investigations menées dans différentes localités ont montré que le nom vernaculaire pouvait correspondre à plusieurs espèces de lavandes mai aussi qu'il pouvait différer d'une lavande à l'autre.

Pour le cas des trois premières espèces L. pedunculata, L. stoechas et L. atlantica, le fait qu'elles portent le même nom devrait paraître normal car même pour les taxonomistes, il y'a toujours eu aussi cette confusion entre les trois, parlant parfois d'espèce L. stoechas avec pedunculata et atlantica deux taxons de rang inférieur (sous espèce) ou encore d'espèces affranchies $L$. pedunculata et $L$. stoechas et seule atlantica est une sous espèce «endémique» de $L$. pedunculata. Pareillement, d'autres travaux, notamment ceux de El Rhaffari (2009), ont rapporté que le nom vernaculaire d'une plante pouvait correspondre à plusieurs espèces à la fois ; tel est le cas du thym « Zaitra» correspondant à Thymus satureioides, $T$. willdenowii, T. pallidus. C'est le cas aussi de l'armoise «Chih» avec Artemisia HerbaHalba, A. Mesatlantica (El Rhaffari, 2009) et de rue «L-Fijel» avec Ruta montana, $R$. graveolens, $R$. chalepensis (Benkhnigue al., 2014). Cette confusion pourrait influencer la qualité du traitement surtout que la composition chimique varie selon les conditions climatiques et édaphiques de la même espèce.

Quant aux trois autres espèces de lavande de cette étude, soient, L. marrocana, L. dentata et L. multifida qui portent chacune un nom local propre, cela est en faveur d'un grand degré de précision chez les riverains surtout que la ressemblance morphologique est très grande entre L. marrocana et $L$. multifida et pourtant ils réussissent à les différentier l'une de l'autre.

Toujours est- il, afin d'éviter toute confusion, l'identification de toute lavande doit être faite par des spécialistes, sur la base de critères taxonomiques, notamment, la feuille, l'inflorescence, la bractée, le calice et la corolle. Aussi, il est très important de distinguer clairement entre les différentes lavandes, surtout si les investigations phytochimiques et le dosage des principales familles chimiques venaient à révéler des différences qualitatives et/ou quantitatives.

Les usages de la plante selon les localités

La lavande est traditionnellement utilisée comme plante aromatique, culinaire, décorative, cosmétique et médicinale (Maganga, 2004).

Le présent travail a de son côté révélé que la lavande est utilisée essentiellement en phytothérapie ; beaucoup d'auteurs ont aussi rapporté que la lavande est utilisée en aromathérapie pour traiter les dépressions, la fatigue et l'hypertension et aussi dans la préparation des parfums, les savons, les poudres de talc et les bougies parfumées (Chu et kemper, 2001). 
Parties de la plante utilisées et modes de préparation

Les parties végétales utilisées pour usage thérapeutique diffèrent selon les espèces et les localités ; d'après notre enquête, il ressort que l'usage des feuilles et des fleurs ensemble est plus fréquent $(72 \%)$ et l'utilisation des fleurs et des feuilles séparément reste relativement faible.

En outre, la partie aérienne des lavandes reste la plus utilisée, en rapport avec la facilité de récolte et les résultats thérapeutiques bénéfiques rapportés expliquent que les principes actifs recherchés sont stockés dans cet organe.

D'une part, dans les différentes localités, la majorité des remèdes est préparée sous forme de décoction. Ce mode reste le moyen le plus efficace qui permet l'extraction et l'assimilation des principes actifs tout en réchauffant le corps et en désinfectant la plante ; cependant, il pourrait détruire certains principes actifs des espèces utilisées (Benlamdini et al., 2014).

En outre, des spécificités d'usage liées à chaque localité persistent ; cela traduit d'une part l'originalité et la diversité des traditions thérapeutiques et leur inhérence aux populations locales; de plus, il serait très intéressant d'investir dans ce volet pour voir si derrière cette différence de modes de préparation des remèdes à base de lavandes entre les localités et / ou entre les espèces il y aurait une différence de composition chimique et d'efficacité de traitement.

\section{-Les pathologies traitées}

Les résultats de cette étude ont montré que, les différentes espèces de lavande sont utilisées essentiellement pour des vertus thérapeutiques.

Selon Bellakhder (2006) la lavande dentée est indiquée essentiellement dans le traitement des affections gastro-intestinales et broncho-pulmonaire. Le décocté de la partie aérienne de Lavandula multifida est utilisé contre les affections pulmonaires (El Rhaffari, 2009), ce qui est en accord avec les résultats obtenus dans la présente investigation ethnobotanique.

D'autre part, les sommités fleuries de Lavandula stoechas sont utilisés comme remède contre les affections pulmonaires (Gülçin et al., 2004), alors que l'infusion de la partie aérienne de la même espèce est utilisée comme antidouleurs (Schulz et al., 2005). Cela concorde avec notre étude, où au niveau de la localité d'Oulmès, région relativement humide et froide en hiver, le rhumatisme est la pathologie la plus traitée par la lavande stéchade utilisée en l'occurrence comme antiinflammatoire.

Encore une fois, apparaît la précision de la pharmacopée traditionnelle marocaine ; en effet, quoique l'on soit dans la même région (cas du plateau central) et que les lavandes soient très affines morphologiquement ( $L$. stoechas et $L$. atlantica), il y a une nette différence dans leurs usages respectifs. De la même façon, en haut Atlas, les deux lavandes ( $L$. dentata et $L$. maroccana), cette fois-ci nettement différenciées quant à leur morphologie, n'ont aucun usage thérapeutique en commun.

\section{Conclusion}

Les prospections floristiques ont permis d'identifier sur la base essentielle de critères morphologiques, différentes espèces de lavande spontanées au Maroc et de dévoiler leurs exigences climatiques et édaphiques. De leur côté, les investigations ethnobotaniques menées auprès d'herboristes et de vendeurs de lavandes dans les régions où celles-ci furent récoltées ont révélé les principaux usages de ce type de plantes, notamment en thérapie, pour le traitement d'affections bronchopulmonaires, gastro-intestinales et urogénitales.

\section{REMERCIEMENTS}

Nous remercions sincèrement les herboristes et vendeurs de plantes aromatiques et médicinales et le personnel des eaux et forêts et des directions provinciales d'agriculture dans les différentes régions 
concernées par la présente étude qui ont apporté leur aide pour l'accomplissement de ce travail.

\section{REFERENCES}

Bellakhdar J. 2006. Plantes Médicinales au Maghreb et Soins de Base. Le Fennec : Casablanca.

Benabdelkader T. 2014. Biodiversité, bioactivité et biosynthèse des composes terpéniques volatils des Lavandes Ailées, Lavandula stoechas Sensu Lato, un complexe d'espèces méditerranéennes d'intérêt pharmacologique, thèse en Biologie et Ecophysiologie Végétale de l'Ecole Normale Supérieure de KoubaAlger, Algérie et de l'Université JeanMonet de Saint-Etienne, France, p259.

Benlamdini N, Elhafian M, Rochdi A, Zidane L. 2014. Étude floristique et ethnobotanique de la flore médicinale du Haut Atlas oriental (Haute Moulouya). $J$. Appl. Biosci., 78: 6771-6787.

Benkhnigue O, Ben Akka F, Salhi S, Fadli M, Douira A, Zidane L. 2014. Catalogue des plantes médicinales utilisées dans le traitement du diabète dans la région d' $\mathrm{Al}$ Haouz-Rhamna (Maroc). Journal of Animal \&Plant Sciences, 23: 2071-7024.

Chu CJ, Kemper KJ. 2001. Lavender (Lavandula spp.). Longwood Herbal Task Force; $32 \mathrm{p}$.

Dupont F, Guignard JL. 2012. Abrégés de pharmacie. Botanique, les familles de plantes. $\left(15^{\mathrm{e}}\right.$ édn). Elsevier Masson : Paris.

EL Rhaffari L, Zaid A .2004. Pratique de la phytothérapie dans le sud-est du Maroc (Tafilalet). Un savoir empirique pour une pharmacopée rénovée. Origine des pharmacopées traditionnelles et élaboration des pharmacopées savantes, Edition de l'Institut de Recherche pour le Développement : Paris ; 293-318.

El Rhaffari. L. 2009. Plante Aromatique et Médicinale de haut atlas Oriental. Edition UCODEP.

Gulcin I, Sat IG, Beydemir S, Elmastas M, Kufrevioglu OI. 2004. Comparison of antioxidant activity of clove (Eugenia caryophylata Thunb) buds and lavender (Lavandula stoechas L.). Food Chemistry, 87: 393-400.

Hmamouchi M. 2001. Les Plantes Médicinales et Aromatiques Marocaines ( $2^{\mathrm{e}}$ edn).

Kitwa AY, Malebo HM. 2004. Malaria control in Africa and the role of traditional medicine. In Traditional Medecinal Plants Abd Malaria. Wilcox M, bodeker G, Rasoanaivo P. CRC Press: boca Raton, FL; 3-18.

Lis-Balchin M. 2002. History of nomenclature of Lavandula species, hybrids and cultivars. In Lavender: the Genus Lavandula (1ère edn). Thaylor et Francis: London; 51-56.

Maganga A. 2004. Influence of variety and organic cultural pratices on yield and essential oil content of Lavender and Rosemary in Interior BC. (STOPA). Ecorational Technologies Kamloops. BC, p 23.

Schulz H, Ozkan G, Baranska M, Kruger H, Ozcan M. 2005. Characterisation of essential oil plants from Turkey by IR and Raman spectroscopy, Vib. Spectrosc. 39(2): 249-256.

Upson T, Andrews S. 2004. The Genus Lavandula. Portland and Oregon. Timber Press: USA. 